\section{Desempeño académico en el primer año de medicina: relación con intereses vocacionales, actitud ante el estudio y fuerza de motivación}

Michell Montalvo-Aguilar, José Luis Armenta-Velázquez, Francisco Gutiérrez-Méndez, Iván Bazán-De Santillana, Israel Castañeda-Andrade, Virginia G. Chávez-Montes de Oca, María de los Ángeles Acevedo-Marrero, Enrique Sánchez-Valdivieso

Introducción. Factores muy diversos, entre ellos los motivacionales, son determinantes para decidir estudiar medicina: el agrado por el ámbito académico y las ciencias básicas, la inclinación hacia la investigación científica y la aspiración a un alto nivel social y económico. Se cree que las características personales, familiares, sociodemográficas y el perfil de intereses y motivaciones de los estudiantes de medicina ejercen influencia en su perspectiva profesional. Influye también el parentesco con médicos y el contacto frecuente y temprano con la medicina, así como el grado de educación de los padres.

Sujetos y métodos. Como parte del examen de admisión se aplicó a los estudiantes un examen de ubicación. Durante el primer semestre se aplicó un cuestionario de fuerza de motivación para la Escuela de Medicina (SMMS) y después se obtuvo el aprovechamiento del primer año.

Resultados. Se evaluó a 63 alumnos. Se encontró una relación de tener bajo aprovechamiento con la obtención de una puntuación menor en las áreas de intereses vocacionales y de razonamiento, así como una mayor tendencia a presentar bajo nivel organizacional y bajo nivel de satisfacción.

Conclusiones. Este estudio permite conocer, en gran medida, cómo influyen en el desempeño académico factores intrínsecos como la perspectiva que el estudiante tiene de la carrera, los resultados de la evaluación psicomotivacional, el interés vocacional y su compromiso social. Identificando deficiencias y detectando problemas se pueden implementar soluciones para mejorar el proceso de la educación médica.

Palabras clave. Desempeño académico. Educación. Intereses vocacionales. Medicina. Motivación.

Academic performance in the first year of Medicine and its relationship to vocational interests, attitude toward study and strength of motivation

Introduction. A number of factors, including motivation, determine decision to study medicine: liking for the academic and basic science, the inclination towards scientific research and the quest for a high social and economic status. It is believed that personal, family, and socio-demographic characteristics, along with interest profile and motivations influence medical students in their professional perspective. It is also influenced by a relative doctor, an early contact with medicine, and higher level of education of parents.

Subjects and methods. As part of the school admission exam, a placement test was applied to the student. During the first semester students completed the questionnaire Strength of Motivation for Medical School (SMMS) and we then got their academic marks for the first year.

Results. Sixty-three students were tested. Relationship was found in having low achievement and getting a lower score in the areas of vocational interests and reasoning and more likely experience low organizational level and low level of satisfaction.

Conclusions. This study showed how student perspective about the medical career, results of his/her psycho-motivational test, vocational interests, and degree of social commitment influence the students' academic performance. Identifying gaps and detecting problems solutions can improve the process of medical education.

Key words. Academic performance. Education. Medicine. Motivation. Vocational interests.
Departamento de Investigación. Universidad Cristóbal Colón. Boca del Río, Veracruz, México.

Correspondencia:

Prof. Enrique Sánchez Valdivieso. Departamento de Investigación. Escuela de Medicina y Escuela de Psicología. Universidad Cristóbal Colón. Ctra. Veracruz-Medellín, s/n 94271 Boca del Río, Veracruz, México.

E-mail:

coordinacion.d.investigacion@ gmail.com

Agradecimientos:

A los siguientes profesores, por sus interesantes sugerencias $y$ comentarios: A. Alexander Aguilera, V. Bernal Dolores, S. Cruz Duarte,

S. Gómez Dorantes, E. González

de la O, F. González Velázquez,

A. Palestino Vilchis, M.A. Regalado

Torres, C. Urbina y J. Valerio Ureña.

Conflicto de intereses:

No declarado.

Competing interests:

None declared.

(C) 2013 FEM 


\section{Introducción}

Evolucionamos hacia una enseñanza dirigida a que el alumno aprenda a ser crítico de la información y a que los profesores centren sus estrategias de acuerdo con los intereses, motivaciones y estilos de aprendizaje de sus alumnos. El estudiante elige la carrera de medicina por intereses de tipo altruista y humanitario [1]. Lo que los atrae son los ideales [2]. La fuerza motriz que motiva a las personas incide en todas las acciones del ser humano, deriva de una jerarquía de necesidades, afecta la elección de la carrera, siendo de dos tipos: la intrínseca (realizar una actividad por la satisfacción que uno experimenta mientras aprende) y la extrínseca (influencias externas al individuo, como los familiares médicos de primer grado). El género frecuentemente influye en la conducta vocacional del sujeto [3]. La fuerza de motivación se define como la disponibilidad del estudiante para iniciar y continuar su educación médica independientemente de sacrificios, infortunios o perspectivas desalentadoras [4]. Sin embargo, a pesar de que los estudiantes pueden llegar con niveles muy altos de motivación, es muy frecuente que decaiga con el paso de los años [5]. La Escuela de Medicina de la Universidad Cristóbal Colón es una institución privada de reciente creación. En un estudio previo [6] hemos confirmado que si bien el alumno comienza la carrera con una fuerza de motivación marcada, dicha fuerza disminuye al finalizarla. El objetivo de este estudio fue evaluar la influencia de la motivación en el desempeño académico de los estudiantes de medicina de primer año y si ello se asocia con el interés vocacional. Conocer si los intereses vocacionales se asocian con la fuerza de motivación para estudiar medicina y si esto podría garantizar un buen desempeño académico sería de gran valor para plantear una estrategia de intervención.

\section{Sujetos y métodos}

Como parte del examen de admisión a la carrera, se sometió a los estudiantes al examen de ubicación [7-11]. Como métodos empíricos aplicados a la muestra se utilizaron, además, un cuestionario de datos generales, la encuesta de fuerza de motivación y, al final del año lectivo, se obtuvo el promedio de cada uno de los estudiantes. El examen de ubicación consta de tres áreas: una área de intereses vocacionales, una de razonamiento y una de actitudes ante el estudio. Las puntuaciones en cada una de las diferentes áreas y subáreas serían: bajas, 1-30; medias, 31-70, y altas, 71-100.

\section{Área de intereses vocacionales}

El inventario de intereses vocacionales mide el grado de atracción o inclinación que tiene el alumno hacia el desarrollo de tareas específicas, elemento importante para descubrir su vocación. El inventario comprende diez subáreas de interés, de las cuales se seleccionaron las tres siguientes en los estudiantes de medicina:

- Área científico-biológica (ACB). Mide el interés por manifestar placer investigando la razón de las cosas, la naturaleza de las causas y cómo resolver problemas de distinta índole en relación a los seres vivos.

- Área verbal (AVB). Mide el interés por la lectura, la oratoria o cualquier otro tipo de actividad que requiera la comprensión y la expresión verbal, tanto oral como escrita.

- Área de servicio social (ASE). Mide el interés por realizar obras de beneficio social preocupándose por el bienestar del ser humano y buscando resolver problemas de su comunidad.

\section{Área de razonamiento}

Mide la habilidad mental de razonamiento verbal, numérico, lógico y espacial (se excluyó del análisis en nuestros estudiantes la prueba de razonamiento numérico por considerarse fuera del perfil):

- Razonamiento verbal. Mide la capacidad de comprensión y uso del lenguaje, análisis, síntesis, relación de ideas y formación de conceptos. Una puntuación alta se relaciona con un buen desempeño de tareas que requieran lenguaje de manera más elaborada, y una puntuación baja, con dificultades para la comprensión y uso del lenguaje, tanto verbal como escrito: análisis, exposición, conclusiones, argumentación y discusión.

- Razonamiento lógico. Mide la capacidad de pasar de unas proposiciones a otras, de lo conocido a lo menos conocido. Una puntuación alta se asocia con capacidad de utilizar los diferentes tipos de razonamiento (deductivo, inductivo), tareas que requieran funciones lógicas de manera más compleja, y una puntuación baja, con dificultad para manejar el orden entre objetos y para tareas como inferencia, organización y conclusión.

- Razonamiento espacial. Mide la capacidad de relacionar una secuencia geométrica en un espacio tridimensional, mediante mecanismos perceptivo-visuales y memoria gráfica y representativa. Con una puntuación alta se espera la construcción de figuras mentales, la aplicación de intuición geométrica, cálculo matemático-espacial 
y elaboración de dibujos y figuras en tres dimensiones. Con una puntuación baja, dificultad para imaginar el objeto en diferentes orientaciones, para lectura de mapas y ubicación en un espacio, y para la previsualización de tamaños y formas.

\section{Área de actitudes ante el estudio}

La tercera área mide la actitud ante el estudio, tomando en cuenta las reacciones emocionales del alumno frente al trabajo escolar. Estudiamos las siguientes escalas en el estudiante de medicina:

- Ansiedad. Expresa la inquietud que le provoca el trabajo mental, partiendo de exigencias excesivas, imaginarias o reales, para pensar, sentir y actuar en formas particulares (reacción ante la presión).

- Obsesión. Estado en el que el alumno mantiene el esfuerzo hasta terminar una tarea (perseverancia) pese a obstáculos (oposición o desaliento).

- Organización. Capacidad de disponer, estructurada e integralmente, de los diferentes elementos necesarios para el logro de una actividad.

- Motivación. Impulso del estudiante que da dirección a su trabajo, orientándolo hacia la consecución de un resultado.

- Desequilibrio somático. Capacidad de controlar o manejar saludablemente las respuestas corporales producidas por la presión escolar (respuesta física al estrés).

- Satisfacción. Identificación de las propias competencias académicas que conducen al reconocimiento del logro y a un sentimiento de satisfacción personal.

\section{Procedimientos adicionales}

En el transcurso del primer semestre se aplicó un cuestionario para medir la fuerza de motivación para la Escuela de Medicina (SMMS), validado por nuestro grupo, con 16 preguntas tipo Likert y que consta de una parte indicativa (nueve preguntas) y una parte contraindicativa (siete preguntas). Para las preguntas indicativas se asigna la puntuación marcada por el estudiante y para cada pregunta contraindicativa la puntuación marcada se invierte. Las puntuaciones máxima y mínima posibles son 80 y 16, respectivamente. Después se obtuvo del registro escolar el promedio del primer año de cada uno de los alumnos.

Finalmente se compararon los resultados del examen de ubicación según el aprovechamiento escolar en dos grupos seleccionados para ello: en el grupo A se seleccionaron 14 alumnos con los más bajos promedios (estudiantes 'en riesgo'; promedio: $7,0)$ y en el grupo B se incluyeron 8 alumnos con los promedios más altos (promedio: 9,0).

\section{Resultados}

Resultaron inscritos en el primer año de la carrera de Medicina de la Universidad Cristóbal Colón 71 alumnos, de los cuales 33 eran mujeres y 38 varones, con una edad promedio de 18,7 años (mediana: 18 años). Informaron vivir con ambos padres el 38,7\%, con uno solo de los padres el $21 \%$, en residencias universitarias el $21 \%$ y vivían solos el 19,3\%. El 30,7\% eran hijos de médicos, el 6,5\% trabajaba y el 3,2\% tenía hijos. Provenían de fuera de la ciudad 33 alumnos (seis de ellos de otro estado); los 38 restantes afirmaron vivir en la conurbación Veracruz-Boca del RíoMedellín. Se excluyeron ocho estudiantes que no completaron todo el proceso, por lo que se evaluó a 63 alumnos, que constituyen la base del estudio.

\section{Área de intereses vocacionales}

- $A C B$. Todos los encuestados dieron puntuaciones mayores de 75 (promedio: 93,3; rango: 78-100), y el $74,2 \%$, una puntuación mayor de 91 .

- $A V B$. El 82,5\% de los alumnos obtuvo una puntuación $\leq 68$ (en dos tercios de estos alumnos la puntuación fue $\leq 40$ ).

- ASE. El 74,6\% de los estudiantes obtuvo una puntuación $\leq 68$.

Resulta llamativa una puntuación baja $(\leq 30)$ en el $27 \%$ del total en el AVB y el 23,8\% del total en el ASE.

\section{Área de razonamiento}

- Razonamiento verbal. El 55,5\% de los alumnos obtuvo menos de 30 puntos (afectación de la capacidad para realizar tareas del orden de la argumentación y discusión).

- Razonamiento lógico. El 61\% de los estudiantes obtuvo menos de 30 puntos (poco desarrollo de la habilidad para utilizar este tipo de razonamiento).

- Razonamiento espacial. Se observó una puntuación media-alta en el 69,9\% (y en las puntuaciones medias era evidente una tendencia hacia puntuaciones altas).

\section{Área de actitudes ante el estudio}

- Ansiedad. Sólo una minoría de estudiantes presentó más de 70 puntos $(17,5 \%)$, lo que indica que 
Tabla I. Distribución de los porcentajes según puntuación alta en los grupos de aprovechamiento.

\begin{tabular}{|c|c|c|c|}
\hline & & Grupo A $(n=14)$ & Grupo B $(n=8)$ \\
\hline \multirow{3}{*}{$\begin{array}{l}\text { Área de intereses } \\
\text { vocacionales }\end{array}$} & Científico-biológica & Promedio: 94,9 & Promedio: 90,3 \\
\hline & Verbal & $7,1 \%$ & $12,5 \%$ \\
\hline & Servicio social & $8,6 \%$ & $25,0 \%$ \\
\hline \multirow{3}{*}{$\begin{array}{l}\text { Área intelectual } \\
\text { y razonamiento }\end{array}$} & Razonamiento verbal & $21,4 \%$ & $75,0 \%$ \\
\hline & Razonamiento lógico & $42,9 \%$ & $87,5 \%$ \\
\hline & Razonamiento espacial & $14,3 \%$ & $62,5 \%$ \\
\hline \multirow{6}{*}{$\begin{array}{l}\text { Área de actitud } \\
\text { ante el estudio }\end{array}$} & Ansiedad & $42,9 \%$ & $50,0 \%$ \\
\hline & Obsesión & $57,1 \%$ & $62,5 \%$ \\
\hline & Organización & $57,1 \%$ & $100 \%$ \\
\hline & Motivación & $57,1 \%$ & $62,5 \%$ \\
\hline & Desequilibrio & $57,1 \%$ & $62,5 \%$ \\
\hline & Satisfacción & $7,1 \%$ & $50,0 \%$ \\
\hline
\end{tabular}

los estudiantes poseen un regular-buen manejo de la presión, es decir, utilizan adecuados recursos de afrontamiento.

- Obsesión. La mayoría de los estudiantes entrevistados $(60,3 \%)$ presentó una puntuación mayor de 70 . El restante $39,7 \%$ obtuvo una puntuación media.

- Organización. El 65,1\% tuvo más de 70 puntos, es decir, podía organizar de una manera efectiva los elementos necesarios para el logro de más de una actividad.

- Motivación. Una puntuación mayor de 70 en la mitad de los estudiantes y una puntuación media (31-70) en el resto, lo que significa que los estudiantes mantienen el impulso hasta la consecución de sus objetivos con poca o nula dificultad.

- Desequilibrio somático. El 63,5\% de alumnos obtuvo 30 puntos o menos en esta prueba. Sin embargo, el $15,9 \%$ obtuvo más de 70 puntos y un adicional 20,6\%, una puntuación media, pero con tendencia hacia una puntuación alta. Ante ciertas situaciones de descontrol, entonces la tensión podría manifestarse incluso físicamente en el $36,5 \%$ de los alumnos.

- Satisfacción. Es muy llamativo que un 82,5\% de los estudiantes encuestados tenía una baja satisfacción (puntuaciones de 30 o menos), con un adicional $15,9 \%$ con puntuaciones medias.

\section{Cuestionario SMMS}

No se observaron correlaciones significativas de la fuerza de motivación con el área de intereses vocacionales o de razonamiento.

\section{Relación entre la fuerza de motivación y las actitudes ante el estudio}

La única correlación (rho de Spearman) que puede considerarse como 'baja' fue en relación a la obsesión. Sin embargo, el valor sólo demuestra una tendencia y no permite hipotetizar posibles asociaciones.

\section{Relación entre la fuerza de motivación y el desempeño académico}

El promedio de aprovechamiento fue de 7,7. Se encontró sólo una correlación espuria (rho de Spearman, $p=0,069)$ de la fuerza de motivación con el aprovechamiento del primer año de la carrera.

\section{Comparación de alumnos destacados y alumnos 'en riesgo'}

Se comparó un grupo de 14 alumnos (grupo A) con bajo aprovechamiento escolar (estudiantes 'en riesgo', promedio: 7,0) con otro grupo de ocho estudiantes (grupo B) con los promedios más altos de la generación (promedio: 9,0). Si bien los alumnos del grupo A tenían una puntuación promedio ligeramente mayor en el ACB respecto al grupo B $(94,9$ frente a 90,3), sólo el 7,1\% y el 8,6\% de los alumnos del grupo A obtuvo una puntuación mayor de 70 en el AVB (grupo B: $12,5 \%$ ) y en el ASE (grupo B: $25 \%$ ), respectivamente.

Como se aprecia en la tabla I, parece existir una relación entre tener un promedio bajo y una menor AVB, ASE y organización, así como menor puntuación en el área de razonamiento (como un predictor de bajo aprovechamiento) y una mayor tendencia a presentar bajo nivel organizacional y bajo nivel de satisfacción.

\section{Relación entre los intereses vocacionales, AVB y ASE y desempeño académico} ¿Una puntuación baja en el AVB se asocia con una puntuación baja también en el ASE? Para rechazar la hipótesis nula de independencia se dividió arbitrariamente a los estudiantes en altos y bajos según si habían obtenido una puntuación mayor o menor a 50 puntos en el ASE y el AVB, se analizaron dichos valores mediante la prueba de chi al cuadrado y se encontró una asociación estadística extremadamente significativa (test exacto de Fisher, dos colas; $p<0,0001$ ) (Tabla II). Obtener una puntuación baja $(\leq 50)$ en una de dichas áreas es predictor de 
una puntuación baja en la otra área. Los alumnos con menos de 50 puntos en el ASE (12 mujeres y 17 varones) en su mayoría presentaban menos de 50 puntos en el AVB (11 mujeres y 14 varones, con cuatro excepciones). Los alumnos con puntuación en $\mathrm{ASE} \geq 51$ y en $\mathrm{AVB} \leq 50$ presentaron significativamente mejores promedios en el sexo masculino (promedio en varones: 8,0 ) en comparación con los alumnos con $\geq 51$ puntos tanto en el ASE como en el AVB (promedio en varones: 7,15).

Entonces se subdividió a todos los alumnos en dos grupos según la puntuación obtenida en el ACB. Cuando obtuvieron más de 96 puntos en el ACB (grupo 1) también presentaban puntuaciones más elevadas en el ASE y $\leq 40$ puntos en el AVB (sólo cuatro estudiantes tuvieron puntuaciones altas en el ASE y el AVB). En los estudiantes con puntuaciones mayores de 51 en el AVB y el ASE, el promedio fue de 7,6 $(n=10)$, y en aquellos con puntuación $\leq 50$ sólo en el AVB, el promedio fue de 8,0 $(n=4)$. En el grupo $2(n=13)$ se colocaron los alumnos que tuvieron 81-95 puntos en el ACB y $\geq 51$ puntos tanto en el ASE como en el AVB, los cuales obtuvieron un promedio de 8,0 (en este grupo se incluyó el alumno con el promedio más elevado). En el grupo 3 $(n=21)$ se colocaron los que tenían 81-95 puntos en el ACB pero $\leq 50$ puntos tanto en el ASE como en el AVB; estos alumnos tuvieron un promedio de 7,7 (Tabla III).

Por tanto, parecería tolerarse mejor tener puntuaciones bajas en el AVB. Además, estudiantes con puntuaciones $\leq 30$ en el ASE tuvieron un mal desempeño académico, pero fue menos evidente cuando en el AVB obtuvieron $\leq 47$ puntos. Así mismo, a los estudiantes con una puntuación $\leq 40$ en el AVB también les fue mal, excepto cuando en el ASE tuvieron una puntuación $\geq 34$.

\section{Motivación}

La mayoría de alumnos comunicó una fuerza de motivación de aceptable a alta (65\%) siendo, en un estudiante adicional, muy alta. La cuarta parte de alumnos informó de una fuerza de motivación de baja a regular, y sólo cuatro alumnos comunicaron una fuerza de motivación muy baja (Tabla IV).

\section{Discusión}

El idealismo consiste en perseguir propósitos nobles, es el servicio a la gente necesitada y la preocupación por la sociedad como un todo [5]. Sin embargo, después del primer año de carrera, hay cambios de las actitudes de los estudiantes que impactan
Tabla II. Distribución de los estudiantes según puntuaciones en las áreas verbal y de servicio.

\begin{tabular}{llll}
\hline & & \multicolumn{2}{c}{ Área verbal } \\
\cline { 3 - 4 } & & $\leq 50$ puntos $(n=35)$ & $\geq 51$ puntos $(n=28)$ \\
\hline Área de servicio & $\leq 50$ puntos & 25 (11 mujeres y 14 varones) & 4 (1 mujer y 3 varones) \\
\cline { 2 - 4 } & $\geq 51$ puntos & 10 (7 mujeres y 3 varones) & 24 (12 mujeres y 12 varones) \\
\hline
\end{tabular}

Tabla III. Puntuaciones promedio en las dos áreas y los diferentes grupos según el área científico-biológica (ACB).

\begin{tabular}{lccc}
\hline & $\begin{array}{c}\text { Grupo 1 } \\
(\mathrm{ACB}>96)\end{array}$ & $\begin{array}{c}\text { Grupo 2 } \\
(\mathrm{ACB}=81-95)\end{array}$ & $\begin{array}{c}\text { Grupo 3 } \\
(\mathrm{ACB}=81-95)\end{array}$ \\
\hline Área de servicio & 72,2 & 56,7 & 32,8 \\
\hline Área verbal & 71,9 & 39,7 & 27,3 \\
\hline Promedio & 7,6 & 8,0 & 7,7 \\
\hline
\end{tabular}

Tabla IV. Distribución de los alumnos según su fuerza de motivación.

\begin{tabular}{|c|c|c|c|}
\hline & Fuerza de motivación & Alumn & \\
\hline$\geq 77$ puntos & Muy alta & $1(2 \%)$ & \\
\hline $72-76$ puntos & Alta & 14 & \multirow{2}{*}{35 (65\%) } \\
\hline $67-71$ puntos & Aceptable & 21 & \\
\hline $62-66$ puntos & Regular & 9 & \multirow{2}{*}{$14(26 \%)$} \\
\hline 57-61 puntos & Baja & 5 & \\
\hline$\leq 56$ puntos & Muy baja & $4(7 \%)$ & \\
\hline
\end{tabular}

negativamente sobre las dimensiones humanísticas del médico [12-14]. Es de primordial importancia atender no sólo a los componentes cognitivos implicados en el aprendizaje del alumno, sino también a los de carácter afectivo $[15,16]$. Las características personales y sociodemográficas y el perfil motivacional ejercen una poderosa influencia sobre las decisiones del estudiante. Factores motivacionales como tener familiares médicos, el agrado por el ámbito académico, la inclinación hacia la investigación científica y la aspiración a un alto nivel económico y social facilitan la identificación y el despertar del interés por la profesión.

La motivación de llegar a ser médico se relaciona con la energía y los sacrificios que el estudiante 
esté dispuesto a invertir. Se ha informado que los distintos sexos se acercan a estudiar medicina desde supuestos diferentes y con motivaciones distintas $[4,12,17,18]$. El desempeño académico, medido por el aprovechamiento escolar, puede estar influido en gran medida por el factor motivacional, como mecanismo para lograr objetivos determinados [19]. Los estudiantes motivados aprenden con mayor rapidez y más eficazmente que los que no lo están [20], siendo el nivel motivacional alcanzado el primer año de estudios un determinante clave de todo el desarrollo de la carrera $[21,22]$. Un bajo rendimiento escolar se relaciona con una baja motivación profesional [23], lo que puede impactar de manera negativa en el desempeño académico. Por ello, se ha recomendado una relación temprana (desde el primer año de la carrera de medicina) del alumno con el paciente y con el hospital como agentes motivacionales [24].

Se ha puesto en duda que exista un grupo común de valores que estén reunidos en cada uno de los integrantes de la profesión médica por su extrema diversificación; sin embargo, a pesar de acoger a individuos con intereses muy dispares, los estudiantes consideran la profesión médica como la única que puede satisfacerlos y dan como importantes razones su interés por el ACB y por el servicio a los demás [1]. En el área de intereses vocacionales existe una correlación directa entre el gusto por el ACB y la elección de los estudios de medicina. A pesar de ello, pocos alumnos tienen claro el espíritu de servicio, ya que es sensiblemente baja la puntuación en el ASE. Debe estimularse dicha área mediante la implementación de estrategias que tiendan a reforzar el trabajo comunitario en zonas marginadas desde etapas tempranas de la carrera.

El 55,5\% de los alumnos presentó afectación de su capacidad para realizar tareas del orden de la argumentación y discusión (puntuación baja en razonamiento verbal). El 61\% de los alumnos tenía poco desarrollada la capacidad de utilizar el razonamiento lógico. Debido a estas bajas puntuaciones es de esperar que los alumnos tengan dificultades en su desempeño académico. Una propuesta sería realizar actividades, dentro de cada materia, tendentes a mejorar tanto el razonamiento verbal y lógico como el AVB. En el área de actitud ante el estudio, los alumnos en general tienen buenas estrategias para enfrentarse a diferentes situaciones escolares, si bien un porcentaje importante tenderá a presentar somatización del estrés.

Se ha demostrado que la motivación influye en el desempeño académico de los estudiantes; una actitud emocional positiva combinada con un grado de satisfacción elevado son indicadores motivacionales que pueden impactar en el desempeño académico. Dos terceras partes de los estudiantes de nuestra escuela manifiestan una fuerza de motivación de aceptable a alta ( $\geq 67$ puntos). Sin embargo, se ha informado que la fuerza de motivación disminuye conforme avanza la carrera, con diferencias dependientes de la edad y el sexo [4]. Aunque el interés vocacional sea positivo para la carrera de medicina, la fuerza de motivación podría considerarse como no suficiente y sería importante investigar qué factores intervienen para que permanezca a la par de los intereses vocacionales.

El desempeño académico del estudiante puede verse influido por sus problemas vocacionales e insatisfacción con la carrera [22], por lo que deben incorporarse estrategias para identificar esas áreas de oportunidad y así retroalimentar tempranamente al estudiante para corregirlas a tiempo. La compatibilidad entre los intereses vocacionales y las características más sobresalientes de la profesión médica, como el idealismo, brindará satisfacción personal al estudiante. Necesitamos conocer el nivel de motivación de los estudiantes por la carrera para poder intervenir de manera efectiva en su formación y en la creación de valores éticos, como parte primordial del proceso docente. Se deben tomar también en consideración variables socioeducativas, como su orientación vocacional y la influencia familiar y social. Se sugiere incluir, en los planes académicos de cada una de las materias del plan de estudios, iniciativas de investigación educativa para desarrollar trabajos que tiendan a detectar deficiencias o instituir soluciones de problemas identificados en el proceso educativo en medicina.

Podemos concluir que existe relación entre un bajo aprovechamiento académico y la obtención de una puntuación baja en las áreas de intereses vocacionales y de razonamiento, y existe también una tendencia a bajos niveles de organización y de satisfacción. Identificar estas tendencias precozmente en el estudiante podría servir para establecer estrategias oportunas con el fin de mejorar el desempeño.

\section{Bibliografía}

1. Soria M, Guerra M, Giménez I, Escanero JF. La decisión de estudiar medicina: características. Educ Med 2006; 9: 91-7.

2. Shaywitz DA, Ausiello DA. Global health: a chance for Western physicians to give and receive. Am J Med 2002; 113: 354-7.

3. Mauleón-Moscardó PJ. Influencias del sexo en las preferencias vocacionales y rasgos de personalidad en los estudiantes de medicina. Actas Esp Psiquiatr 2003; 31: 24-30.

4. Kusurkar R, Kruitwagen C, Ten Cate O, Croiset G. Effects of age, gender and educational background on strength of 
motivation for medical school. Adv Health Sci Educ 2010; 15: 303-13.

5. Smith JK, Weaver DB. Capturing medical students' idealism. Ann Fam Med 2006; 4 (Suppl 1): S32-7.

6. Montalvo AM, Torres HR, Castañeda AI, Chávez MV, Acevedo MA, Sánchez-Valdivieso E. La fuerza de motivación en estudiantes de medicina es dependiente del estrato socioeconómico. Un estudio interuniversitario. URL: http:// www.facmed.unam.mx/sem/jem2010/Abstracts/trabajos_ orales.pdf. [10.04.2012].

7. García-Nieto N. Test TIR (6 VNL). Test ICCE de razonamiento. Madrid: Publicaciones ICCE; 1990.

8. Yuste-Hernanz C. Test THM (6). Test de habilidad mental. Madrid: Publicaciones ICCE; 1991.

9. James A. Test CPE. Cuestionario psicomotivacional de estudio. Madrid: Publicaciones ICCE; 2009.

10. Rivera-Rovirosa AL, Uscanga-Hermida R, Córdoba-Zamudio MA. Test IIVUCC. Inventario de intereses vocacionales de la Universidad Cristóbal Colón. México: Publicaciones ICCE; 2009.

11. Rivera-Rovirosa AL, Rojas-Kramer CA, Córdoba-Zamudio MA Test IPUCC. Inventario de personalidad de la Universidad Cristóbal Colón. México: Publicaciones ICCE; 2009.

12. Roche PW III, Scheetz AP, Dane FC, Parish DC, O'Shea J. Medical student's attitudes in a PBL curriculum: trust, altruism, and cynicism. Acad Med 2003; 78: 398-402.

13. Clever SL, Edwards KA, Feudtner C, Braddock $\mathrm{CH}$ 3rd. Ethics and communication: does students comfort addressing ethical issues vary by specialty team? J Gen Intern Med 2001; 16: 559-63.

14. Roberts LE, Green Hammond KA, Gepperd CM, Warner TD.
The positive role of professionalism and ethics training in medical education: a comparison of medical student and resident perspectives. Acad Psychiatry 2004; 28: 170-82.

15. Huertas JA. Motivación. Querer aprender. Buenos Aires: Aique; 1997.

16. Alonso-Tapia J. Motivar para el aprendizaje. Teoría y estrategias. Barcelona: Edebé; 1997.

17. Coulehan J, Williams P. Vanquishing virtue: the impact of medical education. Acad Med 2001; 76: 598-605.

18. Jordan J, Brown JB, Russell G. Choosing family medicine. What influences medical students? Can Fam Physician 2003; 49: 1065-9.

19. Pérez RM, González CB. Sistema de experto aplicado al rendimiento académico en la enseñanza superior. Educ Med Super 1998; 12: 57-63.

20. Míguez M. El núcleo de una estrategia didáctica universitaria: motivación y comprensión. Revista ieRed 2005. URL: http:// revista.iered.org/v1n3/html/mmiguez.html. [28.09.2009].

21. Durán L. La motivación, factor determinante del éxito. Breves Universidad/Comunidad Escolar 2002; 626: 28-33.

22. Universidad de Chile. Estudio final sobre causas de deserción universitaria. Informe del Centro de Microdatos del Departamento de Economía. Santiago: Universidad de Chile; 2008.

23. Soler $A B$, Chirolde RR. Motivación y rendimiento docente en estudiantes bolivianos del nuevo programa de formación de médicos. Educ Med Super 2010; 24: 42-51.

24. Jorquera-Aguayo P, Acuña-Gamé G, Ramos-Jiménez M. Estrategia de motivación para estudiantes de primer año de medicina, Universidad San Sebastián, Concepción, Chile. Educ Med 2010; 13: 97-100. 